17.05

\title{
In vivo Assessment of the Human Nigrostriatal Dopaminergic System Using Positron Emission Tomography
}

\author{
K.L. Leenders
}

Paul Scherrer Institute, PET Department, Villigen, CH-5232, Switzerland

Direct biochemical functional analysis of implanted cells in human brain during life is not possible. However, one can obtain specific regional cerebral biochemical information using special radiotracer techniques and positron emission tomography (PET). The information carriers are tracer substances, labelled with short-lived positron emitting radionuclides (e.g. ${ }^{11} \mathrm{C}$ or ${ }^{18} \mathrm{~F}$ ), which, after administration to healthy volunteers or patients, are distributed in space and time according to their pharmacokinetic properties. Comparison of the time course of count rates in brain regions with specific tracer uptake to those in brain reference regions allows quantification of specific tracer uptake in terms of metabolic trapping or binding to receptor sites. Quantification may be attempted through application of rigorous mathematical kinetic models or via simple but robust measures like ratios of count rates between two brain regions.

Several PET radiotracers now exist to investigate various aspects of the dopaminergic nigrostriatal neurotransmitter system. Pathophysiological changes at the level of the striatum can be detected and followed longitudinally, or pharmacodynamic interactions with drugs can be studied.

$6-\left[{ }^{18} \mathrm{~F}\right]$-Fluoro-L-dopa is an L-dopa analogue which is specifically trapped in the dopaminergic nigrostriatal nerve terminals after decarboxylation to the amine form. $6-\left[{ }^{18} \mathrm{~F}\right]$-Fluoro-L-dopa uptake is thus a measure of striatal dopaminergic nerve terminal decarboxylation capacity, which at the same time parallels nerve terminal dopamine storage capacity. This tracer does not measure endogenous dopamine concentration, since kinetically it is not exchanging with that pool. However, 6- $\left[{ }^{18} \mathrm{~F}\right]$-fluoro-L-dopa uptake does mimic exogenous L-dopa uptake into the brain both qualitatively and quantitatively. In Parkinson's disease $6-\left[{ }^{18} \mathrm{~F}\right]$-fluoro-L-dopa uptake is decreased in striatum, particularly in putamen (40-50\% of control values) and to a lesser degree in caudate nucleus $(80 \%$ of controls).

$\left[{ }^{11} \mathrm{C}\right]$-Raclopride, a dopamine-D2 antagonist, binds selectively to dopamine-D2 receptors which are, for the most part, post-synaptically located on striatal projection neurons. In early stage Parkinson's disease we have found increased specific dopamine-D2 receptor binding in putamen $(20 \%)$ in proportion to the severity of clinical signs and symptoms. Probably this correlates directly with the presynaptic dopaminergic deficit as assessed by $6-\left[{ }^{18} \mathrm{~F}\right]$ fluoro-L-dopa.

The pattern of uptake of $6-\left[{ }^{18} \mathrm{~F}\right]$-fluoro-Ldopa and $\left[{ }^{11} \mathrm{C}\right]$-raclopride in putamen and caudate nucleus in Parkinson's disease contrasts with that seen in other neurodegenerative disorders.

The effect of striatal transplantation of tissue in patients with dopaminergic neurotransmitter damage can be investigated by comparing preand post-operative PET tracer uptake using the above-mentioned dopaminergic radiotracers.

PET scans after autologous adrenal medulla implantation into striatum neither showed changes in dopamine-D2 receptor binding in two patients using the tracer $\left[{ }^{11} \mathrm{C}\right]$-raclopride (Ann Neurol 1987; 22: 457-468), nor changes in 6$\left[{ }^{18} \mathrm{~F}\right]$-fluoro-L-dopa or $\left[{ }^{11} \mathrm{C}\right]$-nomifensine uptake in two other patients (personal results, unpublished). One study on 5 patients also did not find significant changes of $6-\left[{ }^{18} \mathrm{~F}\right]$-fluoro-L-dopa uptake after implantation (Can J Neurol Sci 16:305-309). All the patients mentioned above 
did not show or only transiently showed clinical improvements.

PET scans on patients who received fetal dopaminergic neurons have been systematically performed in only a few cases. The results on 4 patients have been extensively published (Arch Neurol 1989; 46: 615-631; Science 1990; 247: 174-177; Ann Neurol 31: 155-165 and 166-173). Mainly 6- $\left[{ }^{18} \mathrm{~F}\right]$-fluoro-L-dopa was used as a tracer in these studies. Patients 1 and 2 (implants in putamen and caudate nucleus in one hemisphere) only mildly improved clinically and showed only little concomitant improvement of $6-\left[{ }^{18} \mathrm{~F}\right]$-fluoro-L-dopa uptake on the sites of implantation. Patients 3 and 4 (implanted only in putamen on one side) were operated using improved techniques of tissue preparation and implantation. They showed marked clinical improvement over the course of several months paralleled by a significantly increased $6-\left[{ }^{18} \mathrm{~F}\right]-$ fluoro-L-dopa uptake on the site of the implanted putamen. This tracer increase was main- tained over a period of 3 years after operation. At the same time the tracer uptake in the nonoperated sites showed a progressive decrease in these two patients.

Two severely affected MPTP-induced parkinsonian patients have also been investigated using PET before and after bilateral fetal grafting into putamen and caudate nucleus (Rest Neurol Neurosc 1992; 4(3): 181). PET 6-[ ${ }^{18}$ F]-fluoro-Ldopa scans showed a marked increase of uptake at 1 year after operation concomitant with significant improvement in motor function.

Although the total number of patients studied to date has of necessity been small, these subjects were carefully selected, thoroughly investigated and followed-up. The degree of survival and outgrowth of fetal cells after striatal implantation is associated with a corresponding improvement of $6-\left[{ }^{18} \mathrm{~F}\right]$-fluoro-L-dopa uptake on the implanted sites. PET applied in this way thus provides an effective means to check the success of implantation procedures. 

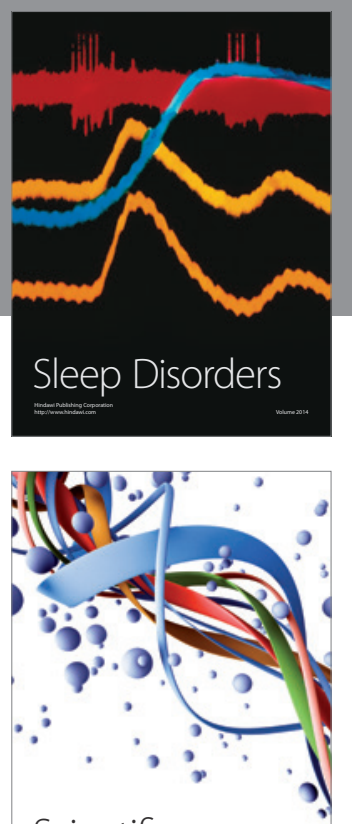

Scientifica
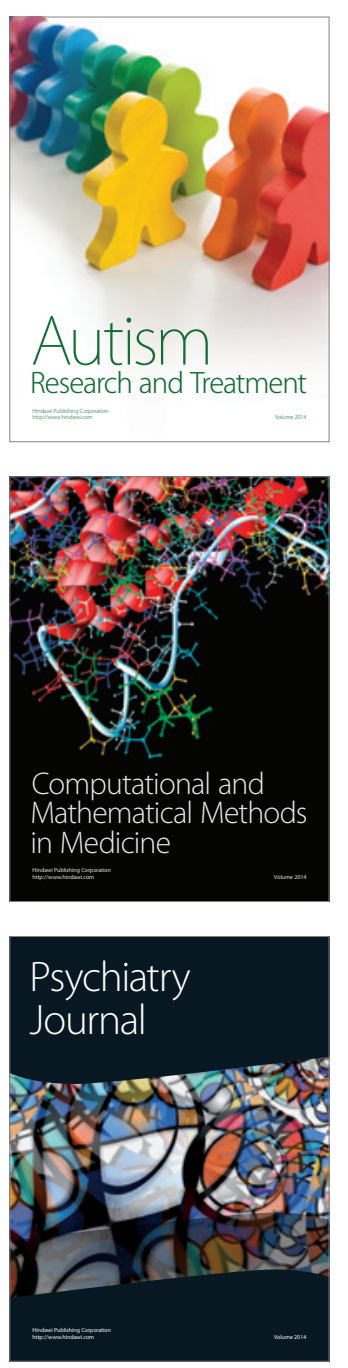
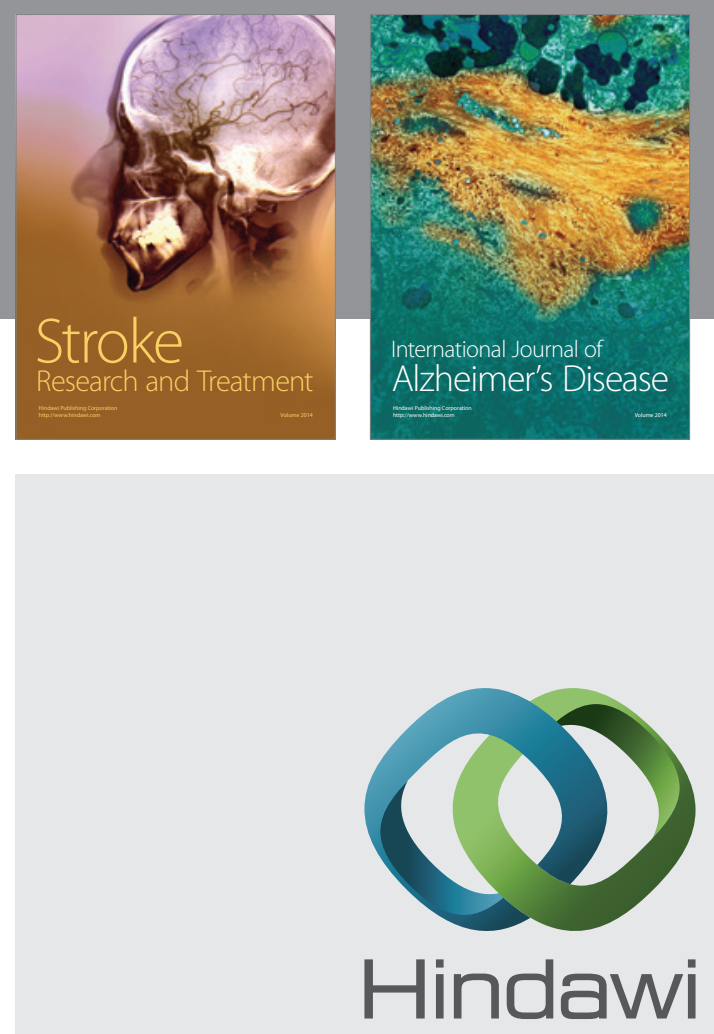

Submit your manuscripts at

http://www.hindawi.com
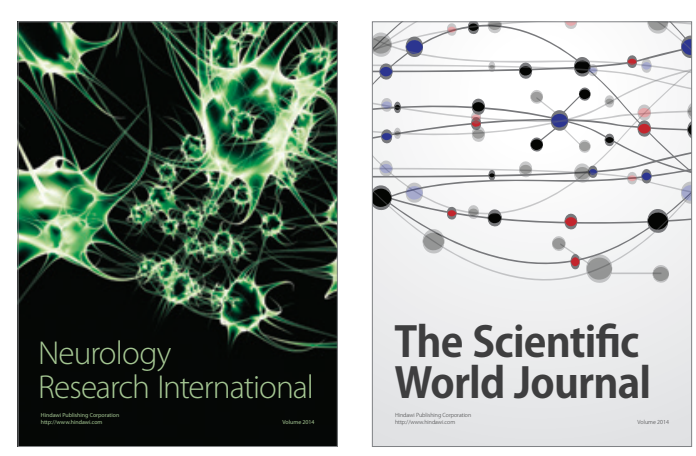

The Scientific World Journal

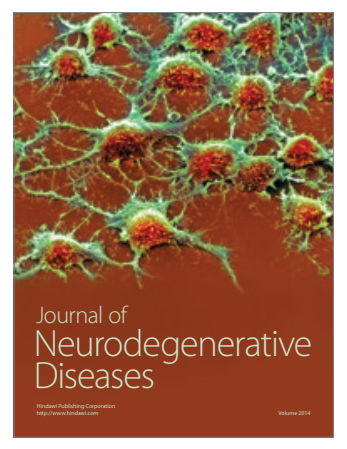

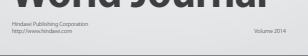

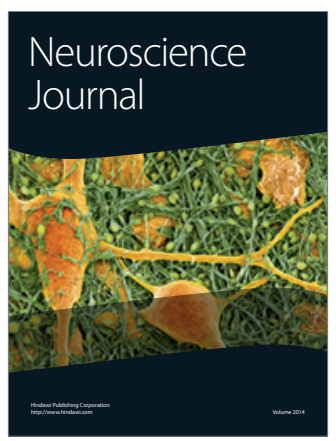

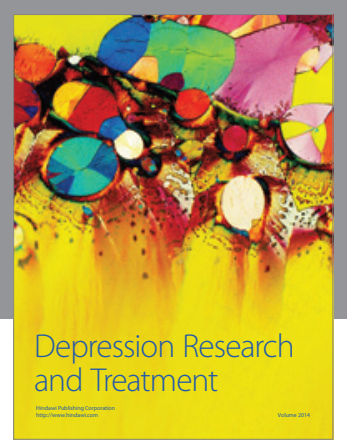
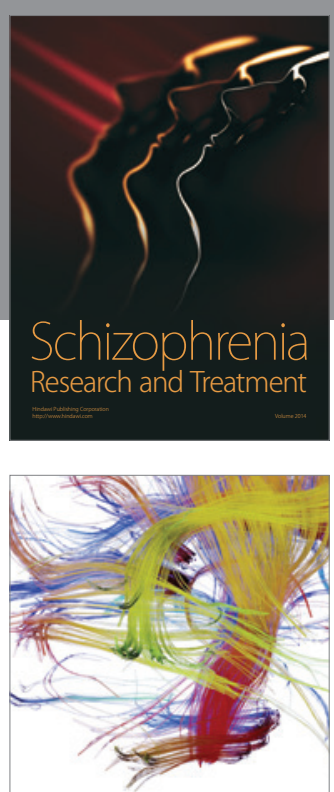

Brain Science

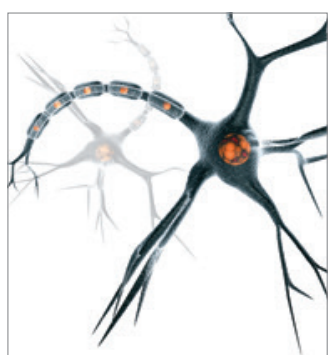

Neural Plasticity
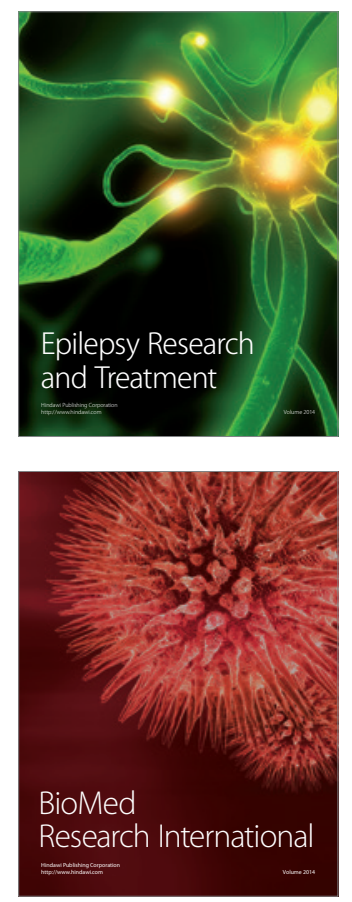

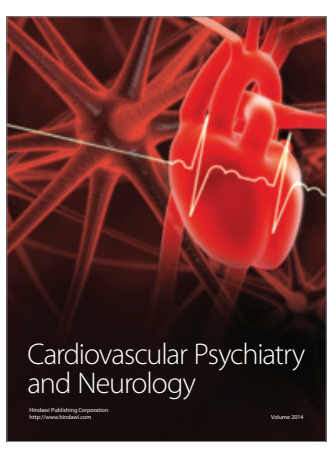

Parkinson's

Disease
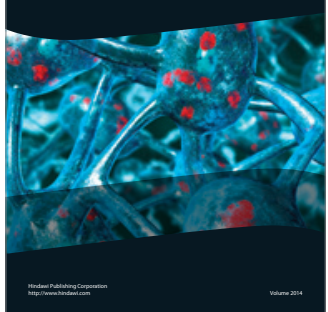